\title{
Chronic Obstructive Pulmonary Disease and Obstructive Sleep Apnoea
}

\author{
Walter T McNicholas
}

Department of Respiratory and Sleep Medicine, St Vincent's University Hospital, School of Medicine, University College Dublin, Dublin, Ireland

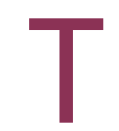

he overlap syndrome (OS) of chronic obstructive pulmonary disease (COPD) and obstructive sleep apnoea (OSA) is common but often missed in clinical practice. Different clinical COPD phenotypes influence the likelihood of co-existing OSA with the predominant emphysema phenotype being protective whereas the predominant chronic bronchitis phenotype promotes the development of OSA. The management of OS differs from COPD alone, particularly the use of nocturnal positive airway pressure (PAP), and patients with OS not treated with PAP have a worse prognosis, which underlines the importance of correct diagnosis.

DOl: https://doi.org/10.17925/ERPD.2017.03.01.23

\section{Keywords}

COPD, sleep apnoea, overlap syndrome, mechanisms, phenotypes, management

Disclosure: Walter T McNicholas has no conflicts of interest to declare in relation to this article. This article is a short opinion piece and has not been submitted to external peer reviewers. No funding was received in the publication of this article.

Authorship: All named authors meet the International Committee of Medical Journal Editors (ICMJE) criteria for authorship of this manuscript, take responsibility for the integrity of the work as a whole, and have given final approval for the version to be published.

open Access: This article is published under the Creative Commons Attribution Noncommercial License, which permits any non-commercial use, distribution, adaptation and reproduction provided the original author(s) and source are given appropriate credit.

Received: 20 February 2017

Published Online: 14 April 2017

Citation: European Respiratory \& Pulmonary Diseases, 2017;3(1):23-4

Corresponding Author: Walter T McNicholas, Department of Respiratory and Sleep Medicine, St Vincent's University Hospital, Elm Park, Dublin 4, Ireland. E: walter.mcnicholas@ucd.ie
For two such highly prevalent disorders, the co-existence of chronic obstructive pulmonary disease (COPD) and obstructive sleep apnoea (OSA) in the same patient gets remarkably little attention in routine clinical practice. Recent estimates indicate that up to $50 \%$ of adult males in the general population have sleep-disordered breathing based on an apnoea-hypopnoea frequency (AHI) over five events per hour, although the prevalence of a clinically significant disorder based on associated symptoms is considerably lower. ${ }^{12} \mathrm{COPD}$ is also highly prevalent, with population estimates of $10 \%$ for COPD associated with significant airflow obstruction. ${ }^{3}$ Thus, by chance alone, COPD and sleepdisordered breathing can be predicted to occur in about $3-4 \%$ of the general adult population, with COPD and a clinically significant OSA syndrome occurring in 1-2\%. In considering the relationships between COPD and OSAS, several questions can be considered. For example, does the presence of either COPD or OSA increase the likelihood of the other disorder in an individual patient? Does the presence of both disorders together, termed 'overlap syndrome', increase the likelihood of co-morbidities? And what are the implications of overlap syndrome for patient management and prognosis?

Regarding COPD predisposing to OSA, one has to consider the influence of different COPD phenotypes. In particular, the emphysema phenotype that is typically associated with lung hyperinflation and low body mass index (BMI) is likely to be protective against OSA, ${ }^{5,6}$ whereas the chronic bronchitis phenotype that is associated with chronic productive cough, higher BMI, and predisposition to cor pulmonale is more likely to predispose towards OSA. ${ }^{7}$ Recent evidence indicates that lung hyperinflation is associated with reduced pharyngeal collapsibility, ${ }^{5}$ and $\mathrm{AHI}$ is negatively correlated with the degree of emphysema on computed tomography (CT) of the thorax in COPD patients. ${ }^{6}$ Peripheral oedema in patients with cor pulmonale predisposes to OSA as a result of rostral fluid shift during the night associated with the supine position, contributing to narrowing of the oropharynx by fluid build-up. ${ }^{8}$ The evidence that OSA contributes to COPD is less clear, although there is some epidemiological evidence that both COPD and asthma are more prevalent in patients with OSA, but the mechanisms of this association have not been established. ${ }^{9}$

Concerning co-morbidities, COPD and OSA are both recognised as independent risk factors for cardiovascular co-morbidity, ${ }_{10,11}$ and it appears reasonable to postulate that patients with overlap syndrome will be at greater risk of co-morbidity. However, there have been remarkably few reports in the literature regarding the risk of cardiovascular disease in patients with overlap syndrome. Overlap patients demonstrate more pronounced hypoxaemia during sleep than patients with either COPD or OSA alone and therefore they are more prone to develop pulmonary hypertension. ${ }^{12}$ Both COPD and OSA alone are associated with evidence of systemic inflammation, ${ }^{13,14}$ which is postulated as an important mechanism in the development of cardiovascular and metabolic disease. However, there have been very few studies that have assessed measures of systemic inflammation or other basic mechanisms that may contribute to cardiometabolic disease in patients with overlap syndrome. 
The diagnosis of OSA requires a combination of clinical assessment and overnight sleep studies, ${ }^{15}$ which represents a more complex assessment than required for the diagnosis of COPD. Thus, clinical awareness is important in the recognition of possible OSA in COPD patients and screening questionnaires may assist in this regard. ${ }^{16}$ Furthermore, since overlap patients are more likely to be hypoxaemic and hypercapnic, particularly during sleep, related clinical features such as morning headache and peripheral oedema as a result of cor pulmonale are likely to be more prevalent in patients with the overlap syndrome. Diagnostic sleep studies in patients with suspected OSA increasingly rely on limited cardiorespiratory measurements, frequently performed in the patient's home, which represents a more cost-effective approach to the diagnosis than the traditional approach of full polysomnography (PSG) in the sleep laboratory. ${ }^{17}$ However, since sleep quality is generally poor in patients with COPD, ${ }^{18}$ PSG studies may be preferable in selected patients, particularly those reporting poor sleep quality. However, where resources are limited, overnight oximetry may be sufficient in many patients, particularly using an oximeter that provides a printed reading that allows identification of the typical transient dips in oxygen desaturation that characterise obstructive apnoea.

The recognition of OSA in COPD patients has important clinical relevance, as the management of overlap syndrome is significantly different than COPD alone, particularly in the use of non-invasive pressure support during sleep, usually in the form of continuous positive airway pressure (CPAP). Furthermore, recent evidence indicates that patients with overlap syndrome treated with CPAP have significantly improved survival compared to those not treated with $\mathrm{CPAP}^{19}$ which indicates that failure to recognise co-existing OSAS in patients with COPD is likely to result in worse survival. Thus, many questions remain open regarding the epidemiology, clinical consequences and outcomes of patients with overlap syndrome, and this topic remains an important and clinically relevant subject for future research. $\square$
1. Heinzer R, Vat S, Marques-Vidal P, et al., Prevalence of sleepdisordered breathing in the general population: the HypnoLaus study, Lancet Respir Med, 2015;3:310-8.

2. Marti-Soler H, Hirotsu C, Marques-Vidal P, et al., The NoSAS score for screening of sleep-disordered breathing: a derivation and validation study, Lancet Respir Med, 2016;4:742-8.

3. Diaz-Guzman E, Mannino DM, Epidemiology and prevalence of chronic obstructive pulmonary disease, Clin Chest Med, 2014;35:7-16.

4. MCNicholas WT, Chronic obstructive pulmonary disease and obstructive sleep apnea: overlaps in pathophysiology, systemic inflammation, and cardiovascular disease, Am I Respir Crit Care Med, 2009;180:692-700.

5. Biselli P, Grossman PR, Kirkness JP, et al., The effect of increased lung volume in chronic obstructive pulmonary disease on upper airway obstruction during sleep, J App/ Physiol, 2015;119:266-71.

6. Krachman SL, Tiwari R, vega ME, et al., Effect of emphysema severity on the Apnea-Hypopnea Index in smokers with obstructive sleep apnea, Ann Am Thorac Soc,
2016;13:1129-35.

Ustun B, Westover MB, Rudin C, Bianchi MT, Clinical prediction models for sleep apnea: the importance of medical history over symptoms, J Clin Sleep Med, 2016;12:161-8.

8. Senaratna CV, Perret IL, Lodge CJ, et al., Prevalence of obstructive sleep apnea in the general population: a systematic review, Sleep Med Rev, 2016;34:70-81.

9. Greenberg-Dotan S, Reuveni H, Tal A, et al., Increased prevalence of obstructive lung disease in patients with obstructive sleep apnea, Sleep Breath, 2014;18:69-75.

10. MCNicholas WT, Bonsignore MR, Sleep apnoea as an independent risk factor for cardiovascular disease: current evidence, basic mechanisms and research priorities, Eur Respir J, 2007;29:156-78.

11. Fabbri LM, Luppi F, Beghe B, Rabe KF, Complex chronic comorbidities of COPD, Eur Respir 」, 2008:31:204-12.

12. Chaouat A, Bugnet A-S, Kadaoui N, et al., Severe pulmonary hypertension and chronic obstructive pulmonary disease Am J Respir Crit Care Med, 2005;172:189-94.

13. Ryan S, Taylor CT, MCNicholas WT, Selective activation of inflammatory pathways by intermittent hypoxia in obstructive sleep apnea syndrome, circulation, 2005;112:2660-7.

14. Wouters $\mathrm{E}$, Local and systemic inflammation in chronic obstructive pulmonary disease, Proc Am Thorac Soc 2005;2:26-33.

15. McNicholas WT, Diagnosis of obstructive sleep apnea in adults, Proc Am Thorac SOC, 2008:5:154-60.

16. McNicholas WT, Screening for sleep-disordered breathing: the continuing search for a reliable predictive questionnaire, Lancet Respir Med, 2016;4:683-5.

17. Kuna ST, Badr MS, Kimoff RJ, et al., An official ATS/AASM/ ACCP/ERS workshop report: research priorities in ambulatory management of adults with obstructive sleep apnea, Proc Am Thorac Soc, 2011;8:1-16.

18. McSharry DG, Ryan S, Calverley $P$, et al., Sleep quality in chronic obstructive pulmonary disease, Respirology 2012;17:1119-24.

19. Marin JM, Soriano JB, Carrizo SJ, et al., Outcomes in patients with chronic obstructive pulmonary disease and obstructive sleep apnea, Am J Respir Crit Care Med, 2010:182:325-31. 